\title{
Prevalência de patologias detectadas pela triagem neonatal em Santa Catarina
}

\author{
Prevalence of disorders detected by newborn \\ screening in Santa Catarina
}

Adriana Kleist Clark Nunes', Regiane Gutjahr Wachholz', Marina R. M. Rover', Liliete Canes Souza'

1 Universidade Federal de Santa Catarina (UFSC), Florianópolis, SC, Brasil
Correspondência para: Marina R. M. Rover

Departamento de Análises Clínicas, Centro de Ciências da Saúde, Universidade Federal de Santa Catarina, Campus Universitário 88040-900 - Florianópolis, SC, Brasil marinarover@yahoo.com.br

Recebido em 11/Jul/2012 Aceito em 20/Fev/2013

\section{RESUMO}

Objetivo: Avaliar a prevalência das patologias fenilcetonúria (FNC), hipotireoidismo congênito $(\mathrm{HC})$, fibrose cística $(\mathrm{FC})$, hemoglobinopatias (HB) e hiperplasia adrenal congênita (HAC), no Estado de Santa Catarina, a fim de delinear o perfil da população catarinense em relação a essas patologias. Materiais e métodos: Foi realizado um levantamento de dados do Programa de Triagem Neonatal da Secretaria de Estado da Saúde de Santa Catarina, no período de 2004 a 2008. Resultados: No período de estudo, foram obtidas as seguintes prevalências: FCN 1:28.862, HC 1:2.876, FC 1:5.121, HB S 1:14.446 e para HAC 1:11.655 crianças triadas. Conclusões: A prevalência média da FNC mostrou-se inferior à prevalência nacional, ao passo que a da HAC foi superior; por sua vez, a do $\mathrm{HC}$ mostrou-se semelhante às prevalências mundial e nacional. Além disso, o predomínio da população caucasiana no Estado resultou em uma prevalência reduzida da HB e uma prevalência aumentada da FC em relação ao restante do país. Arq Bras Endocrinol Metab. 2013;57(5):360-7

\section{Descritores}

Triagem neonatal; fenilcetonúria; hipotireoidismo congênito; fibrose cística; hemoglobinopatias; hiperplasia adrenal congênita

\section{ABSTRACT}

Objective: To evaluate the prevalence of the diseases phenylketonuria (PKU), congenital hypothyroidism (CH), cystic fibrosis (CF), hemoglobinopathies (HB), and congenital adrenal hyperplasia (CAH), in the state of Santa Catarina, in order to delineate the local population profile for these diseases. Materials and methods: A survey of data from the Newborn Screening Program of the Ministry of Health of Santa Catarina, in the period 2004 to 2008 was carried out. Results: During the study period, the following prevalences were obtained: 1:28,862 children screened for PKU; 1:2,876 children screened for $\mathrm{CH} ; 1: 5,121$ children screened for $\mathrm{CF} ; 1: 14,446$ children screened for HB S; and 1:11,655 children screened for CAH. Conclusions: The prevalence of PKU proved to be lesser than the national prevalence, while $\mathrm{CAH}$ prevalence was greater. On the other hand $\mathrm{CH}$ prevalence was similar to the global and national prevalence. Moreover, the predominance of the Caucasian population in the state resulted in reduced prevalence of $\mathrm{HB} S$ and increased prevalence of CF in relation to the rest of the country. Arq Bras Endocrinol Metab. 2013;57(5):360-7

\section{Keywords}

Neonatal screening; phenylketonuria; congenital hypothyroidism; cystic fibrosis; hemoglobinopathies; congenital adrenal hyperplasia

\section{INTRODUÇÃO}

A triagem neonatal consiste em um exame laboratorial de caráter preventivo que tem por objetivo a detecção precoce de erros inatos do metabolismo e de outras patologias assintomáticas no período neonatal, permitindo a interferência no curso da doença e promovendo, assim, a diminuição ou até mesmo a eliminação das sequelas associadas a cada patologia (1). 
No Brasil, a triagem neonatal teve início em 1976, com o desenvolvimento do projeto de triagem para a fenilcetonúria (FNC) em recém-nascidos, pelo professor Benjamin Schmidt, na Associação de Pais e Amigos dos Excepcionais de São Paulo (Apae-SP) (2). Em 2001, o Ministério da Saúde lançou a Portaria n ${ }^{\circ} 822$, que instituiu, no âmbito do Sistema Único de Saúde, o Programa Nacional de Triagem Neonatal (PNTN), com o objetivo de atender a todos os recém-nascidos em território nacional. O programa tornou-se responsável pela detecção, confirmação, diagnóstico, acompanhamento e tratamento dos casos suspeitos de quatro patologias: FNC, hipotireoidismo congênito (HC), fibrose cística (FC) e hemoglobinopatias $(\mathrm{HB})(3,4)$. Sua implantação nos Estados brasileiros é apresentada de acordo com três fases: Fase I: contempla o diagnóstico de FNC e HC; Fase II: contempla o diagnóstico de FNC, HC e HB; e Fase III: contempla o diagnóstico de FNC, HC, HB e FC. Os laboratórios especializados credenciados pelo PNTN são denominados de Serviços de Referência em Triagem Neonatal (SRTN) e responsáveis pela execução e controle do PNTN em suas áreas de abrangência, devendo haver pelo menos um SRTN em cada Estado brasileiro (1-4). Em maio de 2006, todos os Estados do país já haviam implantado a triagem neonatal para a FNC e o HC, desempenhando suas funções de acordo com os protocolos do PNTN (5) e, atualmente, estão habilitados no PNTN com pelo menos um serviço de referência credenciado pelo Ministério da Saúde (1). Até o ano de 2009, além de Santa Catarina, somente mais três Estados brasileiros (Paraná, Minas Gerais e Espírito Santo) executavam a Fase III do PNTN (1). Entre 2009 e 2011, Goiás, São Paulo, Rio Grande do Sul, Rondônia e Rio de Janeiro passaram para a Fase III (1). Em 2012, mais oito Estados (Bahia, Distrito Federal, Mato Grosso, Pará, Permambuco, Ceará, Maranhão e Mato Grosso do Sul) foram homologados na Fase III do PNTN, perfazendo dezessete Estados, entretanto, como Rondônia realiza a triagem para o Acre, pode-se considerar, efetivamente, dezoito Estados participantes da Fase III (1).

Desde sua implantação em Santa Catarina, a coordenação do programa foi conferida ao Laboratório Central de Saúde Pública (Lacen-SC). No laboratório, as amostras são analisadas e as crianças suspeitas de possuírem doenças neonatais são encaminhadas para o Hospital Infantil Joana de Gusmão (HIJG) para a confirmação do diagnóstico, tratamento e acompanhamento médico, quando necessários. Além da execução dos exames para o diagnóstico das quatro patologias previstas pelo PNTN, o Lacen-SC e o HIJG também são responsáveis pelo diagnóstico precoce da hiperplasia adrenal congênita (HAC), o qual foi incluído na triagem neonatal $(6,7)$.

A FNC é uma doença metabólica hereditária, caracterizada pela ausência ou deficiência quase completa de atividade da enzima fenilalanina hidroxilase, levando ao aumento da concentração de fenilalanina plasmática e da excreção urinária de ácido fenilpirúvico $(5,8)$. Crianças com FNC não tratada apresentam um quadro clínico com sintomas bastante variáveis, incluindo o retardo mental, comportamento altista, convulsões, microencefalia, odor de "rato" da pele, cabelos e urina (em função do acúmulo de fenilacetato), tendência a hipopigmentação e eczema (8-10). No Brasil, os Serviços de Referência em Triagem Neonatal realizam a triagem da FNC a partir da dosagem quantitativa da fenilalanina (FAL) sanguínea, e a obtenção de valores da enzima superiores a $10 \mathrm{mg} / \mathrm{dL}$, em pelo menos duas amostras laboratoriais distintas, fornece o diagnóstico da doença (3).

O HC é a incapacidade da glândula tireoide do recém-nascido em produzir quantidades suficientes dos hormônios tireoidianos e representa uma das principais causas tratáveis de retardo mental. A triagem é feita com dosagens do hormônio estimulante da tireoide (TSH) e da tiroxina (T4) $(3,11)$. Após o resultado positivo inicial no Programa de Triagem Neonatal, devem ser realizadas as dosagens do T4 (total e livre) e do TSH em amostra de sangue venoso, para que haja a efetiva confirmação diagnóstica. Seguindo essa estratégia, a média de detecção dos casos suspeitos atinge em torno de 90\% (3). As crianças não tratadas precocemente terão o crescimento e o desenvolvimento mental comprometidos, ocorrendo manifestações clínicas como: hipotonia muscular, dificuldades respiratórias, cianose, icterícia prolongada, constipação, bradicardia, anemia, sonolência excessiva, livedo reticularis, choro rouco, hérnia umbilical, alargamento de fontanelas, mixedema, sopro cardíaco, dificuldade na alimentação com deficiente crescimento pôndero-estatural, atraso na dentição, retardo na maturação óssea, pele seca e sem elasticidade, atraso de desenvolvimento neuropsicomotor e retardo mental $(3,12)$. Já as crianças que têm o diagnóstico precoce estabelecido pelos Programas de Triagem Neonatal não apresentarão qualquer sintomatologia clínica, desde que a terapia de reposição hormonal seja iniciada no tempo adequado $(3,12)$. 
A FC trata-se da mais comum entre as doenças hereditárias consideradas letais $(3,13)$. As manifestações clínicas da doença consistem no acometimento pulmonar crônico, na insuficiência pancreática e na elevada concentração de cloreto de sódio no suor (14). As manifestações pulmonares resultam de uma intensa reação inflamatória desencadeada por infecções oportunistas nas vias aéreas, fator que gera um dano irreversível ao tecido pulmonar, seguido de óbito por disfunção respiratória (13-15). Os programas de triagem neonatal empregam a dosagem de tripsina imunorreativa (IRT) como o primeiro teste de triagem para a doença, sendo os resultados positivos confirmados por meio da quantificação de cloreto no suor e/ou da análise de DNA $(3,16,17)$.

As HB são doenças hereditárias, de caráter autossômico recessivo, e que resultam de mutações nos genes que codificam as cadeias globínicas alfa $(\alpha)$ e beta $(\beta)$ da molécula de hemoglobina. Afetam, aproximadamente, $7 \%$ da população mundial, sendo as mais frequentes e clinicamente significativas as variantes estruturais para as hemoglobinas S e C, que acometem principalmente populações originárias do Continente Africano, da Região Mediterrânea, do Sudeste Asiático, do Oriente Médio e Extremo Oriente $(18,19)$. As principais complicações clínicas na Anemia Falciforme incluem a anemia hemolítica crônica, episódios dolorosos e intermitentes de vaso-oclusão, risco permanente de infecções como resultado de autoinfarto esplênico, acidentes vasculares cerebrais, retinopatia e danos cumulativos em múltiplos órgãos $(19,20)$. Além da triagem neonatal dessa hemoglobinopatia, o Ministério da Saúde também destaca a importância da detecção precoce da hemoglobinopatia $\mathrm{C}$ e de $\alpha$ e $\beta$ talassemias pelos Programas de Triagem Neonatal (3). A realização do diagnóstico laboratorial precoce dessas alterações hemoglobínicas é de primordial importância, em virtude da alta taxa de mortalidade na infância (2l).

A HAC é um conjunto de defeitos enzimáticos de causa genética, que interfere na biossíntese de cortisol e, frequentemente, de aldosterona. Pode causar a morte nos pacientes com a forma perdedora de sal e também virilização nas mulheres. É caracterizada por defeitos em qualquer uma das enzimas necessárias à síntese do cortisol, e o defeito mais comum é a deficiência da 21-hidroxilase, que pode ser identificada na triagem neonatal pela dosagem da 17-hidroxiprogesterona (22-24). O quadro clínico da HAC é muito variado, depende de haver ou não deficiência de outros esteroides adrenais, do acúmulo dos precursores proximais ao bloqueio enzimático, do desvio do metabolismo adrenal para outras vias metabólicas, e do grau de deficiência enzimática (25). O diagnóstico de HAC mais confiável é a determinação dos valores sanguíneos do precursor do cortisol imediatamente proximal à enzima deficiente. Assim, na deficiência da 21 -hidroxilase, determinam-se as concentrações séricas do precursor da enzima 21-hidroxilase, isto é, a 17-hidroxiprogesterona. Nas crianças com a forma clássica de deficiência da 21-hidroxilase, os valores séricos da 17-hidroxiprogesterona estão alguns graus de magnitude acima dos normais $(26,27)$.

Considerando o exposto, o objetivo deste trabalho foi avaliar a prevalência das patologias FNC, HC, FC, HB e HAC, no Estado de Santa Catarina, a fim de delinear o perfil da população catarinense em relação a essas patologias.

\section{MATERIAIS E MÉTODOS}

O presente estudo foi conduzido com a pesquisa retrospectiva do número de recém-nascidos triados para FNC, HC, FC, HB e HAC, do número de casos cujos diagnósticos foram confirmados para essas patologias e do número de nascidos vivos no Estado de Santa Catarina, no período compreendido entre janeiro de 2004 a dezembro de 2008.

O número de indivíduos triados para FNC, HC, FC, HB e HAC foi obtido por meio do sistema informatizado gerador e gerenciador do banco de dados Vega Triagem $^{\circledR}$ (CCTI, São Paulo, SP), utilizado no setor de Análises Neonatais do Lacen-SC. A busca levou em consideração o número total de crianças triadas, anualmente, pela instituição. O número de casos diagnosticados de FNC, HC, FC, HB e HAC foi confirmado no Setor de Endocrinologia do HIJG. O número de nascidos vivos no Estado de Santa Catarina foi obtido por pesquisa no Sistema de Informações sobre Nascidos Vivos (Sinasc) (28).

A técnica utilizada para a realização dos testes constitui na coleta de sangue capilar em papel-filtro (FITEC $^{\circledR}$ especial com espessura de $2,27 \mathrm{~mm}$, gramatura $105-110 \mathrm{~g} / \mathrm{m}^{2}$ linear 1005 algodão), o sangue foi coletado nos Postos de Saúde, Hospitais ou Maternidades dos municípios participantes do Programa por técnicos de enfermagem treinados, sendo subsequentemente encaminhado para o setor de Análises Neonatais do Lacen-SC. A faixa etária atendida para realização da 
primeira coleta variou de 3 a 14 dias. A FAL (coeficiente de variação intraensaio de $8,5 \%$ e coeficiente de variação interensaio de $5,1 \%$ para valores de $\mathrm{FAL}$ com média de $12,1 \mathrm{mg} / \mathrm{dL}$ ) foi quantificada por imunoensaio no sistema automatizado Wallac 1232 Victor $^{\circledR}$. O TSH (coeficiente de variação intraensaio de $6,8 \%$ e coeficiente de variação interensaio de $8,9 \%$ para valores de TSH com média de 10,5 $\mu \mathrm{UI} / \mathrm{mL}$ ), a IRT (coeficiente de variação intraensaio de $7,1 \%$ e coeficiente de variação interensaio de $8,0 \%$ para valores de IRT com média de $104 \mathrm{ng} / \mathrm{mL}$ ) e a 17-OHP (coeficiente de variação intraensaio de $7,3 \%$ e coeficiente de variação interensaio de $9,6 \%$ para valores de 17 -OHP com média de 16,3 $\mathrm{ng} / \mathrm{mL}$ ) foram quantificados por imunoensaio no sistema automatizado Wallac 1235 AutoDELFIA ${ }^{\circledR}$. As hemoglobinas variantes foram analisadas qualitativamente por cromatografia líquida de alta performance (HPLC - coeficiente de variação intraensaio de $2,2 \%$ para comparações entre bandas de hemoglobina A e C e de 3,3\% entre hemoglobina $\mathrm{A}$ e $\mathrm{F}$ e coeficiente de variação interensaio de 1,8\% para comparações entre bandas de hemoglobina A e C e de 2,9\% entre hemoglobina A e F) em aparelho Bio-Rad Variant ${ }^{\mathrm{TM}}$. Todos os resultados alterados de hemoglobinas foram confirmados com eletroforese em gel de agarose por focalização isoelétrica $\left(\right.$ IEF - Pharmacia Biotech $\left.{ }^{\circledR}\right)$. Os princípios utilizados para análise laboratorial, os analitos determinados na triagem de cada patologia e os respectivos valores de referência utilizados foram citados na tabela 1 . Os valores que estavam acima dos de referência foram repetidos duas vezes com a primeira amostra. Quando necessário, as crianças foram reconvocadas para uma segunda coleta. Com a persistência de valores elevados ou a obtenção de valores muitos elevados já na $\mathrm{l}^{\mathrm{a}}$ amostra, as crianças foram encaminhadas, pelo Lacen-SC, ao Serviço de Endocrinologia Pediátrica do HIJG.

Os critérios a seguir foram utilizados para definição do diagnóstico pelo endocrinologista pediátrico. $\mathrm{Na}$ triagem da FNC, a obtenção, em amostra de sangue total, de valores de FAL (imunoensaio automatizado Wallac 1232 Victor $^{\circledR}$ ) superiores a $10 \mathrm{mg} / \mathrm{dL}$, em pelo menos duas amostras laboratoriais distintas, forneceu o diagnóstico da doença. Para HC foi realizada a dosagem sérica do T4 total $(5,53$ a $11,0 \mu \mathrm{g} / \mathrm{dL})$, do T4 livre $(0,78$ a $2,19 \mathrm{ng} / \mathrm{dL})$ e do TSH $(0,35$ a $5,50 \mu \mathrm{UI} /$ $\mathrm{mL})$ por química seca em sistema automatizado Vitros ${ }^{\circledR}$ ECi (Ortho-Clinical Diagnostics). Assim, o HC foi identificado quando foram obtidos valores de T4 total e livre inferiores a $5,53 \mu \mathrm{g} / \mathrm{dL}$ e $0,78 \mathrm{ng} / \mathrm{dL}$, respectivamente, e valores de TSH superiores a $5,50 \mu \mathrm{UI} / \mathrm{mL}$. Os casos que apresentaram resultados positivos de IRT foram confirmados por meio de uma quantificação de cloreto no suor por fotometria de chama, considerado como padrão-ouro para o diagnóstico confirmatório da $\mathrm{FC}$, sendo que valores desse eletrólito iguais ou superiores a $60 \mathrm{mEq} / \mathrm{L}$ conferem o diagnóstico para a doença. Por constituírem métodos de elevada precisão, HPLC e IEF, todos os resultados positivos foram repetidos para confirmação, e resultados anormais foram confirmados após três meses. Para o diagnóstico de HAC, foram realizadas as dosagens séricas de 17-OHP por radioimunoensaio de 17-alfa-hidroxiprogesterona Coat-A-Count $^{\circledR} \quad$ (Siemens Healthcare Diagnostics), sendo considerados os seguintes valores de referência de acordo com o sexo e a idade: até 1 mês (masculino de 53 a $186 \mathrm{ng} / \mathrm{dL}$, feminino de 17 a $204 \mathrm{ng} / \mathrm{dL}$ ); de $\mathrm{l}$ a 6 meses (masculino de $35 \mathrm{a} 157 \mathrm{ng} / \mathrm{dL}$, feminino de 25 a $110 \mathrm{ng} / \mathrm{dL}$ ); de 6 a 12 meses (masculino de 6 a $40 \mathrm{ng} / \mathrm{dL}$, feminino de 5 a $47 \mathrm{ng} / \mathrm{dL}$ ).

Para análise de dados os valores obtidos foram tabelados em planilha eletrônica de cálculos do Microsoft Office Excel $^{\circledR}$. A cobertura do PNTN em Santa Catarina foi calculada como percentual, considerando-se $100 \%$ o número de nascidos vivos no Estado, relatando-se medidas descritivas de média e desvio-padrão. As prevalências anuais das patologias FC, FNC, HAC, HB

Tabela 1. Exames de triagem neonatal realizados

\begin{tabular}{lccc}
\hline Patologia & Analito analisado & Método de análise & Valores de referência \\
\hline Fenilcetonúria & FAL & Imunoensaio & $<3,00 \mathrm{mg} / \mathrm{dL}$ (sujeitos à reconvocação acima de 2,70) \\
$\begin{array}{l}\text { Hipotireoidismo congênito } \\
\text { Fibrose cística }\end{array}$ & TSH & Imunoensaio & $<10 \mu \mathrm{Ul} / \mathrm{mL}$ \\
& IRT & Imunoensaio & $<70,0 \mathrm{ng} / \mathrm{mL}$ (para detecção de FC o resultado acima de 30 dias de vida \\
não é significativo)
\end{tabular}

HPLC: cromatografia líquida de alta performance, IEF: focalização isoelétrica. 
e HC na população rastreada foram calculadas dividindo-se o número total de crianças triadas pelo número de casos diagnosticados no período avaliado.

O estudo foi delineado de acordo com as Diretrizes e Normas Regulamentadoras de Pesquisa envolvendo Seres Humanos e aprovado pelo Comitê de Ética em Pesquisas com Seres Humanos da Universidade Federal de Santa Catarina (Processo 325, FR 278578).

\section{RESULTADOS}

No período de 2004 a 2008, um total de 378.337 crianças foi triado para as patologias FNC, HC, FC, HB e HAC, pelo setor de Análises Neonatais do Lacen-SC, perfazendo uma média de $75.667 \pm 3.009$ crianças triadas, anualmente, pela instituição. Nesse mesmo período, verifica-se um total de 420.531 nascidos vivos em Santa Catarina, correspondendo a uma média de $84.106 \pm 1.363$ nascimentos anuais, no Estado. No ano de 2005, a triagem neonatal abrangeu grande número de crianças e foi responsável pelo maior percentual do período estudado, equivalente a $94,8 \%$. Para os demais anos os percentuais de cobertura foram: 89,7\% (2004), $89,8 \%$ (2006), $88,4 \%$ (2007) e $87,2 \%$ (2008).

\section{Fenilcetonúria}

O número de casos de FCN totalizou 26 crianças. Esse valor corresponde a uma média de cinco crianças diagnosticadas, anualmente, com a doença e resulta em uma prevalência média para o período estudado de 1:28.862 na população rastreada. De acordo com a tabela 2 , observa-se uma grande variação da prevalência da FNC na população de estudo, elevando-se de 1:76.631, em 2004, para 1:7.369, em 2008, valores justificados pelo aumento de dez casos confirmados no ano de $2008 \mathrm{em}$ relação ao ano de 2004.

Tabela 2. Total de crianças triadas e diagnosticadas para a FNC e prevalência da doença na população rastreada, no período de 2004 a 2008

\begin{tabular}{|c|c|c|c|c|}
\hline & $\begin{array}{l}\text { Número de } \\
\text { crianças } \\
\text { triadas para } \\
\text { a FCN }\end{array}$ & $\begin{array}{l}\text { Falso- } \\
\text { positivos na } \\
\text { segunda } \\
\text { coleta }\end{array}$ & $\begin{array}{l}\text { Casos } \\
\text { confirmados } \\
\text { de FCN }\end{array}$ & $\begin{array}{c}\text { Prevalência } \\
\text { de FCN na } \\
\text { população } \\
\text { rastreada }\end{array}$ \\
\hline 2004 & 76.631 & 15 & 1 & $1: 76.631$ \\
\hline 2005 & 80.146 & 2 & 6 & $1: 13.358$ \\
\hline 2006 & 75.571 & 0 & 7 & $1: 10.796$ \\
\hline 2007 & 72.312 & 3 & 2 & $1: 36.156$ \\
\hline 2008 & 73.677 & 0 & 10 & $1: 7.368$ \\
\hline
\end{tabular}

\section{Hipotireoidismo congênito}

O número de casos de $\mathrm{HC}$ totalizou 132 crianças. Esse valor corresponde a uma média de 26 crianças diagnosticadas, anualmente, com a doença e resulta em uma prevalência média de 1:2.876 triados, na população estudada. De acordo com a tabela 3 , a prevalência de $\mathrm{HC}$ na população rastreada manteve-se relativamente constante no período de 2004 a 2008 , variando entre l:3.065 a 1:2.781 triados, nos anos de 2004 e 2007, respectivamente.

Tabela 3. Total de crianças triadas e diagnosticadas para o $\mathrm{HC}$ e prevalência da doença na população rastreada, no período de 2004 a 2008

\begin{tabular}{ccccc}
\hline & $\begin{array}{c}\text { Número de } \\
\text { crianças } \\
\text { triadas para } \\
\text { o HC }\end{array}$ & $\begin{array}{c}\text { Falso- } \\
\text { positivos na } \\
\text { segunda } \\
\text { coleta }\end{array}$ & $\begin{array}{c}\text { Casos } \\
\text { confirmados } \\
\text { de HC }\end{array}$ & $\begin{array}{c}\text { Prevalência } \\
\text { de HC na } \\
\text { população } \\
\text { rastreada }\end{array}$ \\
\hline 2004 & 76.631 & 0 & 25 & $1: 3.065$ \\
2005 & 80.146 & 0 & 28 & $1: 2.862$ \\
2006 & 75.571 & 0 & 29 & $1: 2.605$ \\
2007 & 72.312 & 0 & 26 & $1: 2.781$ \\
2008 & 73.677 & 0 & 24 & $1: 3.069$ \\
\hline
\end{tabular}

\section{Fibrose cística}

O número de casos de FC totalizou 76 crianças no período de 2004 a 2008. Esse valor corresponde a uma média de 15 crianças diagnosticadas anualmente com a doença e resulta em uma prevalência média de 1:5.121 na população rastreada. De acordo com a tabela 4 , a prevalência de FC na população rastreada não apresentou grandes flutuações, variando de 1:3.684, em 2008, para 1:6.165, em 2005.

Tabela 4. Total de crianças triadas e diagnosticadas para a FC e prevalência da doença na população rastreada, no período de 2004 a 2008

\begin{tabular}{ccccc}
\hline & $\begin{array}{c}\text { Número de } \\
\text { crianças } \\
\text { triadas para } \\
\text { a FC }\end{array}$ & $\begin{array}{c}\text { Falso- } \\
\text { positivos na } \\
\text { segunda } \\
\text { coleta }\end{array}$ & $\begin{array}{c}\text { Casos } \\
\text { confirmados } \\
\text { de FC }\end{array}$ & $\begin{array}{c}\text { Prevalência } \\
\text { de FC na } \\
\text { população } \\
\text { rastreada }\end{array}$ \\
\hline 2004 & 76.631 & 32 & 13 & $1: 5.895$ \\
2005 & 80.146 & 0 & 13 & $1: 6.165$ \\
2006 & 75.571 & 0 & 15 & $1: 5.038$ \\
2007 & 72.312 & 58 & 15 & $1: 4.821$ \\
2008 & 73.677 & 69 & 20 & $1: 3.684$ \\
\hline
\end{tabular}

\section{Hemoglobinopatias}

O número de casos de HB S totalizou 24 crianças, das quais 16 apresentaram a hemoglobina variante 
FS e oito crianças apresentaram a hemoglobina FSC, perfazendo uma média de seis crianças diagnosticadas anualmente com a doença. No período de estudo, somente um criança apresentou a hemoglobina variante FC, representando um único caso de HB C confirmado no Estado, que ocorreu no ano de 2008. As prevalências anuais da doença apresentaram-se consideravelmente constantes no período avaliado, variando entre 1:10.947 e 1:18.078 triados (Tabela 5). Dessa forma, a prevalência média da HB S foi de 1:14.446 crianças triadas no período de 2004 a 2008.

Tabela 5. Total de crianças triadas e diagnosticadas para HB e prevalência da doença na população rastreada, no período de 2004 a 2008

\begin{tabular}{lccc}
\hline & $\begin{array}{c}\text { Número de } \\
\text { crianças triadas } \\
\text { para HB }\end{array}$ & $\begin{array}{c}\text { Casos } \\
\text { confirmados de } \\
\text { HB S }\end{array}$ & $\begin{array}{c}\text { Prevalência de HB S } \\
\text { na população } \\
\text { rastreada }\end{array}$ \\
\hline 2004 & 76.631 & 07 & $1: 10.947$ \\
2005 & 80.146 & 06 & $1: 13.357$ \\
2006 & 75.571 & 05 & $1: 15.114$ \\
2007 & 72.312 & 04 & $1: 18.078$ \\
2008 & 73.677 & 05 & $1: 14.735$ \\
\hline
\end{tabular}

\section{Hiperplasia adrenal congênita}

O número de casos com HAC totalizou 77, perfazendo uma média de, aproximadamente, 19 crianças diagnosticadas anualmente com a doença (Tabela 6). Observaram-se no ano de 2008 apenas dois casos da doença diagnosticados, com uma prevalência de 1:36.838, mostrando uma flutuação de valores comparando com as ocorrências de maior número de casos confirmados nos anos anteriores. Assim, a prevalência média calculada para essa patologia no período de 2004 a 2008 foi equivalente a 1:11.655 triados.

Tabela 6. Total de crianças triadas e diagnosticadas para a HAC e prevalência da doença na população rastreada, no período de 2004 a 2008

\begin{tabular}{lcccc}
\hline & $\begin{array}{c}\text { Número de } \\
\text { crianças } \\
\text { triadas para } \\
\text { a FCN }\end{array}$ & $\begin{array}{c}\text { Falso- } \\
\text { positivos na } \\
\text { segunda } \\
\text { coleta }\end{array}$ & $\begin{array}{c}\text { Casos } \\
\text { confirmados } \\
\text { de HAC }\end{array}$ & $\begin{array}{c}\text { Prevalência } \\
\text { de HAC na } \\
\text { população } \\
\text { rastreada }\end{array}$ \\
\hline 2004 & 76.631 & 10 & 15 & $1: 5.108$ \\
2005 & 80.146 & 99 & 23 & $1: 3.484$ \\
2006 & 75.571 & 211 & 30 & $1: 2.519$ \\
2007 & 72.312 & 69 & 07 & $1: 10.330$ \\
2008 & 73.677 & 63 & 02 & $1: 36.838$ \\
\hline
\end{tabular}

\section{DISCUSSÃO}

A partir dos dados obtidos do Programa de Triagem Neonatal foi possível avaliar a prevalência dos distúrbios mais comuns no Estado de Santa Catarina. O presente estudo não contempla as triagens realizadas pela rede de laboratórios privados do Estado, assim, provavelmente, nos anos em que houve um percentual maior de triagens atendidas por esses laboratórios, foi obtida uma cobertura menor pelo Lacen. Nesse contexto, a cobertura do programa no período analisado obteve uma média de $90 \%$, portanto, as prevalências das patologias obtidas a partir da população rastreada podem ser extrapoladas às prevalências encontradas na totalidade da população catarinense. $\mathrm{Na}$ avaliação das patologias triadas, considerando as médias obtidas com os valores dos anos que compõem o período estudado, observou-se que a maior ocorrência foi do HC, com prevalência de 1:2.876, seguido da FC com 1:5.121, da HAC com 1:5.360, da HB S com 1:13.538 e da FCN com prevalência de 1:28.862 na população rastreada.

A média da prevalência do período estudado para a patologia FNC foi inferior à prevalência mundial da doença, a qual corresponde a 1:12.000 nascidos-vivos (29). A prevalência média da doença no Estado mostrou-se também inferior à prevalência nacional, que apresentou valores entre 1:16.691 e 1:24.310 nascidos vivos, nos anos de 2001 e 2002, respectivamente, de acordo com o último levantamento realizado pela Sociedade Brasileira de Triagem Neonatal (30).

A prevalência média do HC em Santa Catarina, no período de 2004 a 2008, mostrou-se semelhante à prevalência mundial da doença, a qual varia em torno de $1: 4.000$ a $1: 3.000$ nascidos vivos (22). Da mesma forma, essa prevalência assemelhou-se à prevalência observada no Brasil, que apresentou valores entre 1:3.694 e 1:3.808 nascidos vivos, nos anos de 2001 e 2002, respectivamente, segundo o último levantamento realizado pela Sociedade Brasileira de Triagem Neonatal (30).

A prevalência de $\mathrm{FC}$ se mostrou semelhante à prevalência mundial da doença, a qual varia de $1: 2.000 \mathrm{a}$ 1:10.000 nascidos vivos de origem caucasiana $(31,32)$. No entanto, mostrou-se um pouco acima da prevalência observada no Brasil, que apresentou valores entre 1:16.275 e 1:13.073 nascidos vivos, nos anos de 2001 e 2002, respectivamente, de acordo com o último levantamento realizado pela Sociedade Brasileira de Triagem Neonatal (30). Essa prevalência aumentada da doença, cuja ocorrência é alta na população de origem caucasiana, ocorre pelo fato de o Estado de Santa Catarina possuir uma maior proporção de indivíduos caucasianos quando comparado com o restante do país. 
No Brasil, destaca-se a alta incidência da HB S, sendo que, de acordo com o Ministério da Saúde, as prevalências da doença em diferentes regiões brasileiras permitem estimar a existência de mais de 8.000 afetados com a forma homozigótica da doença (33). Estima-se que, em determinadas regiões do Brasil, a doença falciforme tenha uma prevalência de 1:333 nascidos vivos (3). Essa afecção tende a ser mais frequente entre indivíduos afro-americanos e hispânicos. Nos Estados Unidos, por exemplo, a prevalência média da doença é de $1: 2.000$ a $1: 2.500$ nascimentos, aumentando para l:400 nascidos vivos na população negra e de 1:500 a 1:1.000 nascidos vivos na população de origem hispânica $(3,22)$. Em Santa Catarina, a anemia falciforme apresentou-se como a mais frequente $\mathrm{HB}$ diagnosticada, totalizando $96 \%$ dos casos. No entanto, a prevalência da doença no Estado, representada pela média de 1:14.446 triados, no período analisado, apresentou-se visivelmente diminuída em relação à prevalência observada no país. Essa prevalência reduzida da doença, cuja ocorrência é maior na população afro-americana, pode ser parcialmente explicada pelo predomínio da população caucasiana no Estado de Santa Catarina, representada por cerca de $90 \%$ dos catarinenses, de acordo com o último censo demográfico realizado no país (34).

A incidência de HAC na população mundial é variável entre diferentes populações, com valores desde de 1:280 (35) a 1:42.000 (36) nascidos vivos, sendo as maiores prevalências verificadas em grupos étnicos específicos (Alasca, Japão e Nova Zelandia). No Brasil, os valores obtidos em diferentes estudos oscilam de 1:7.500 (37) a 1:10.000 nascidos vivos (38). Entretanto, de acordo com o levantamento realizado pela Sociedade Brasileira de Triagem Neonatal, a prevalência da doença variou entre 1:15.460 e 1:17.091, nos anos de 2001 e 2002, respectivamente (30). Assim, considerando-se a prevalência média da forma clássica da HAC em 1:11.655 triados na população catarinense, no período de 2004 a 2008, observam-se valores similares aos verificados em outros estados brasileiros, que também correlacionam com os valores de prevalência mundial.

Estudos nacionais têm mostrado que as coberturas médias dos Programas de Triagem Neonatal variam muito e ainda não atingiram os índices ideais. No Programa de Triagem Neonatal do Hospital das Clínicas da Faculdade de Medicina de Ribeirão Preto, a cobertura está em torno de 94\%, enquanto no Serviço de Referência em Triagem Neonatal no Estado de Mato Grosso a cobertura populacional é inferior a $70 \%(39,40)$.
Em nosso país, embora nos últimos anos uma considerável melhora nos parâmetros de avaliação da qualidade dos programas possa ser vista, esses ainda não são os índices ideais. Campanhas visando à maior divulgação da importância da triagem neonatal são necessárias para aumentar a cobertura, e a instituição do terceiro dia de vida do recém-nascido como sendo o Dia do Teste do Pezinho poderia contribuir para que idades mais precoces de tratamento fossem atingidas, melhorando o prognóstico das crianças acometidas. A idade na coleta e o atraso na fase de confirmação diagnóstica são os principais motivos para o atraso do início do tratamento dos casos detectados pelos serviços.

O Programa de Triagem Neonatal associa tecnologia, conhecimento e informação levando ao diagnóstico precoce, o que permite o controle e a redução de riscos em relação à saúde. Isso leva à redução da morbidade e mortalidade, além de melhor qualidade de vida aos afetados e redução de gastos futuros para a sociedade. Outro benefício da análise neonatal é a identificação dos portadores de algumas patologias, possibilitando o aconselhamento genético aos pais e a possibilidade de um planejamento familiar. É importante enfatizar que a triagem neonatal deve contemplar todos os passos propostos pelo PNTN, inclusive a introdução em tempo hábil do tratamento adequado, para que seus objetivos sejam efetivamente atingidos.

Agradecimentos: aos profissionais do Setor de Análises Neonatais do Laboratório Central da Secretaria de Saúde do Estado de Santa Catarina (Lacen-SC), especialmente as farmacêuticas bioquímicas Lea Ruhland e Igara Edite Noceti e Vieira, pelo auxílio técnico na consulta ao banco de dados. O presente artigo apresenta conteúdo e parte dos resultados do Trabalho de Conclusão de Estágio (Triagem Neonatal pela Secretaria de Estado da Saúde de Santa Catarina: avaliação do período de 1996 a 2008) de Adriana Kleist Clark Nunes e Regiane Gutjahr Wachholz (Curso de Farmácia - Análises Clínicas da UFSC).

Declaração: os autores declaram não haver conflitos de interesse científico neste estudo.

\section{REFERÊNCIAS}

1. Brasil, Ministério da Saúde. O que é triagem neonatal? [citado em 13 Jun, 2012]. Disponível em: http://portal.saude.gov.br/portal/saude/area.cfm?id_area=1061. Acesso em: 17 Dez, 2012.

2. Sociedade Brasileira de Triagem Neonatal. [cited 2009 Jan 15]. Available from: http://www.sbtn.org.br/.

3. Brasil, Ministério da Saúde. Departamento de Atenção Especializada. Manual de normas técnicas e rotinas operacionais do Programa Nacional de Triagem Neonatal. Brasília: Ministério da Saúde; 2004. 
4. Brasil, Ministério da Saúde. Portaria GM/MS n. ${ }^{\circ} 822 / \mathrm{GM}$ em 6 de junho de 2001. Instituição do Programa Nacional deTriagem Neonatal, no âmbito do Sistema Único de Saúde, para fenilcetonúria, hipotireoidismo congênito, fibrose cística e hemoglobinopatias. Brasília: Ministério da Saúde; 2001.

5. Leão LL, Aguiar MJ. Newborn screening: what pediatricians should know. J Pediatr. 2008;84(4 suppl):80-90.

6. Nascimento ML, Pires MM, Nassar SM, Ruhland L. Avaliação do programa de rastreamento neonatal para hipotireoidismo congênito da Secretaria de Estado da Saúde de Santa Catarina. Arq Bras Endocrinol Metab. 2003;47(1):75-81.

7. Mattozo MR, Souza LC. Triagem neonatal em Santa Catarina: relato histórico, aspectos fisiopatológicos e métodos de análise realizados pelo Laboratório Central da Secretaria de Saúde do Estado. Newslab. 2005;68:84-102.

8. Monteiro LTB, Cândido LMB. Fenilcetonúria no Brasil: evolução e casos. Rev Nutr. 2006;19(3):381-7.

9. Mabry-Hernandez I, WolffT, Green K. Screening for phenylketonuria: a literature update for the U.S. Preventive Services Task Force. AHRO Publication. 2008;8:1-5.

10. Vilarinho L, Queirós A, Leandro P, Almeida I, Rivera IT. Fenilcetonúria Revisitada. Arq Med. 2006;20(5-6):161-72.

11. Souza CFM, Schwartz IV, Giugliani R. Triagem neonatal de distúrbios metabólicos. Ciênc Saúde Coletiva. 2002;7(1):129-37.

12. American Academy of Pediatrics. Update of newborn screening and therapy for congenital hypothyroidism. Pediatrics. 2006;17(6):2290-303.

13. Milavetz G, Smith JJ. Cystic Fibrosis In: Dipiro JT, Talbert RL, Yee GC, Matzke GR, Wells BG, Posey LM. Pharmacotherapy: a pathophysiologic approach. 6th ed. New York: McGraw-Hill; 2005. p. 591-603.

14. Rodrigues R, Gabetta CS, Pedro KP, Valderato F, Fernandes MIM, Magalhaes PKR, et al. Cystic fibrosis and neonatal screening. Cad Saúde Pública. 2008;24(4 suppl):475-84.

15. Davis PB. Cystic fibrosis since 1938. Am J Respir Crit Care Med. 2006;173:475-82.

16. Comeau AM, Accurso FJ, White TB, Campbell PW, Hoffman G, Parad RB, et al. Guidelines for Implementation of Cystic Fibrosis Newborn Screening Programs: Cystic Fibrosis Foundation Workshop Report. Pediatrics. 2007;119(2):495-518.

17. Grosse SD, Boyle CA, Botkin JR, Comeau AM, Kharrazi M, Rosenfeld $M$, et al. Newborn Screening for Cystic Fibrosis: Evaluation of Benefits and Risks and Recommendations for State Newborn Screening Programs. Centers for Disease Control and Prevention: Recommendations and Reports; 2004.

18. Sommer CK, Goldbeck AS, Wagner SC, Castro SM. Triagem neonatal para hemoglobinopatias: experiência de um ano na rede de saúde pública do Rio Grande do Sul, Brasil. Cad Saúde Pública. 2006;22(8)1709-14.

19. Sonati MF, Costa FF. The genetics of blood disorders: hereditary hemoglobinopathies. J Pediatr. 2008;84(4 suppl):40-51.

20. Munker R, Mansouri A, Gupte SC, Sakhalkar V. Hemolytic Anemias In: Munker R, Hiller E, Glass J, Paquete R. Modern hematology: biology and clinical management. 2a ed. Totowa: Humana Press; 2007. p. 101-26.

21. Silva KR, Yamaguchi MU. Os benefícios da inclusão das hemoglobinopatias na triagem neonatal. Arq Ciênc Saúde Unipar. 2007;11(1):67-73.

22. Kaye $\mathrm{Cl}$. Committee on genetics. Newborn screening fact sheets. Pediatrics. 2006;118 (3):934-62.
23. Speiser PW, White PC. Congenital adrenal hyperplasia. N Engl J Med. 2003;349(87):776-88.

24. Van der Kamp HJ, Wit JM. Neonatal screening for congenital adrenal hyperplasia. Eur J Endocrinol. 2004;151(3 suppl):71-5.

25. Calliari LEP, Longui CA, Monte O. Endocrinologia para o pediatra. $2^{a}$ ed. São Paulo: Atheneu; 1998.

26. Speiser PW, Azziz R, Baskin LS, Ghizzoni L, Hensle TW, Merke DP, et al.; Endocrine Society. Congenital adrenal hyperplasia due to steroid 21-hydroxylase deficiency: an Endocrine Society clinical practice guideline. J Clin Endocrinol Metab. 2010;95(9):4133-60.

27. Speiser PW, White PC. Congenital adrenal hyperplasia due to 21-hydroxylase deficiency. Endocr Rev. 2000;21:91-245.

28. Ministério da Saúde/FNS/CNEPI/Sistema de Informações sobre Nascidos Vivos - SINASC. [citado em 10 Jun, 2008]. Disponível em: http://www.datasus.gov.br. Acesso em: 17 Dez, 2012.

29. Marton da Silva MBG. A coleta do teste do pezinho deve ocorrer sempre na alta hospitalar. Revista Novidades e Notícias da Associação dos Fenilcetonúricos e Homocistinúricos do Paraná. 2001;5(9):7.

30. Carvalho TM, Pimentel H, Carvalho GPM. Levantamento Epidemiológico Brasileiro da Triagem Neonatal [citado em 16 Jan, 2009]. Disponível em: http://www.sbtn.org.br. Acesso em: 17 Dez, 2012.

31. Faucz FR, Gimenez J, Ramos MD, Pereira-Ferrari L, Estivill $X$, Raskin S, et al. Cystic fibrosis in a southern Brazilian population: characteristics of $90 \%$ of the alleles. Clin Genet. 2007;72:218-23.

32. Santos GPC, Domingos MT, Wittig EO, Riedi CA, Rosário NA. Programa de Triagem Neonatal para Fibrose Cística no Estado do Paraná: avaliação após 30 meses de sua implantação. J Pediatr. 2005;81(3):240-4.

33. Zago MA. Considerações gerais sobre as doenças falciformes. In: Anvisa (Agência Nacional de Vigilância Sanitária). Manual de diagnóstico e tratamento das doenças falciformes. Brasília: Anvisa; 2002. p. 9-11.

34. IBGE. Instituto Brasileiro de Geografia e Estatística. [database on the Internet] [citado em 27 Maio, 2009]. Disponível em: http:// www.ibge.gov.br/home/presidencia/noticias/20122002censo. shtm. Acesso em: 17 Dez, 2012.

35. Pang S, Murphey W, Levine LS, Spence DA, Leon A, LaFranchi S, et al. A pilot newborn screening for congenital adrenal hyperplasia in Alaska. J Clin Endocrinol Metab. 1982;55(3):413-20.

36. Therrell BL Jr, Berenbaum SA, Manter-Kapanke V, Simmank J, Korman K, Prentice $L$, et al. Results of screening 1.9 million Texas newborns for 21-hydroxylase-deficient congenital adrenal hyperplasia. Pediatrics. 1998;101(4 Pt 1):583-90.

37. Pang SY, Clark A. Congenital adrenal hyperplasia due to 21-hydroxylase defieiency: newbom screening and its relationship to the diagnosis and treatment of the disorder. J Med Screen. 1993;2:105-39.

38. Silveira EL, dos Santos EP, Bachega TA, van der Linden Nader I, Gross $\mathrm{JL}$, Elnecave RH. The actual incidence of congenital adrenal hyperplasia in Brazil may not be as high as inferred: an estimate based on a public neonatal screening program in the state of Goiás. J Pediatr Endocrinol Metab. 2008;21(5):455-60.

39. Magalhães PKR, Turcato MF, Angulo IL, Maciel LMZ. Programa de Triagem Neonatal do Hospital das Clínicas da Faculdade de Medicina de Ribeirão Preto, Universidade de São Paulo, Brasil. Cad. Saúde Pública. 2009; 25(2):445-54.

40. Stranieri I, Takano OA. Avaliação do Serviço de Referência em Triagem Neonatal para hipotireoidismo congênito e fenilcetonúria no Estado de Mato Grosso. Brasil. Arq Bras Endocrinol Metab. 2009;53(4):446-52. 